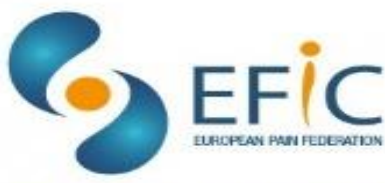

PAIN IN EUROPE X| BRINGING THE FUTURE TO THE PRESENT

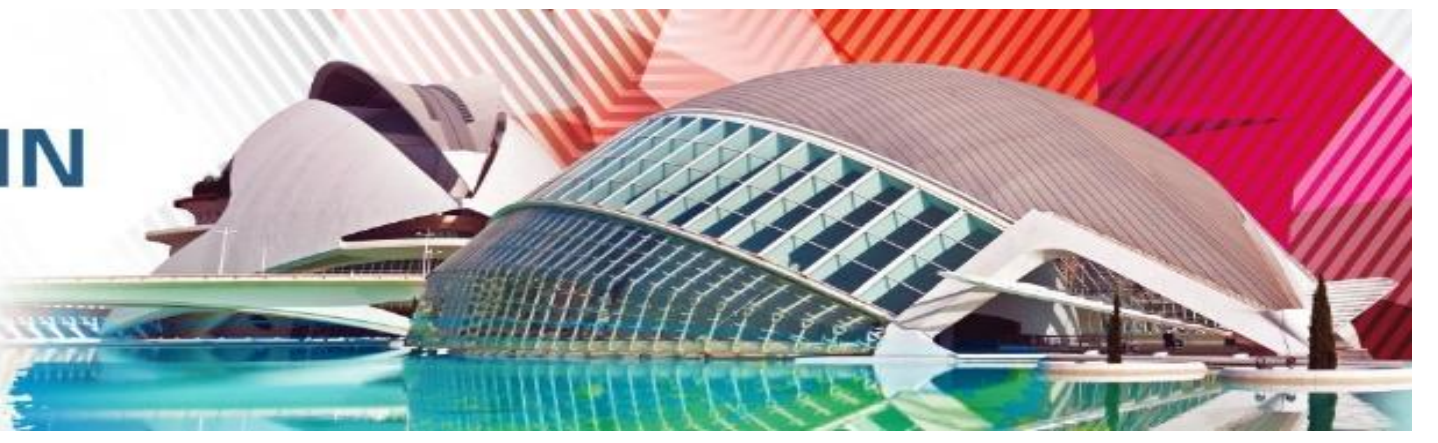

\title{
CHRONIC PAIN IN PRIMARY CARE
}

\section{A PATWAY}

Ana Sofia Vieira Barbosa ${ }^{1}$ Mafalda Cerqueira ${ }^{1}$ Raul Marques Pereira ${ }^{2}$

${ }^{1}$ Training Family physician, ${ }^{2}$ Family physician USF Lethes

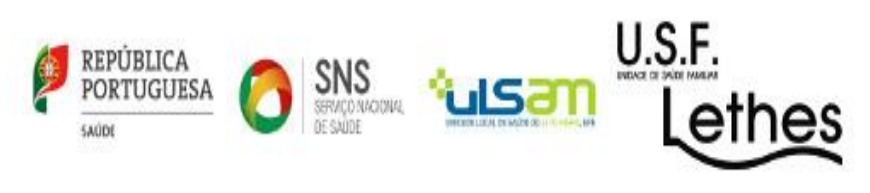

\section{Background and aims}

Last years Chronic Pain has become a scourge of disability for individuals of every age who haven't an adequate response to health care, particularly in Primary Health Care (PHC).

The first step to responding this problem was the creation, in 2015, of a Chronic pain consultation in PHC in the authors' unit.

\section{Methods}

In July 2018, the authors made a review of available evidence to validate a Chronic pain assessment protocol that can be replicated in any Primary Care Unit. The bibliographic research was made through PubMed. The Mesh terms were used: "Chronic Pain" [Mesh] AND "Pain Measurement" [Mesh].

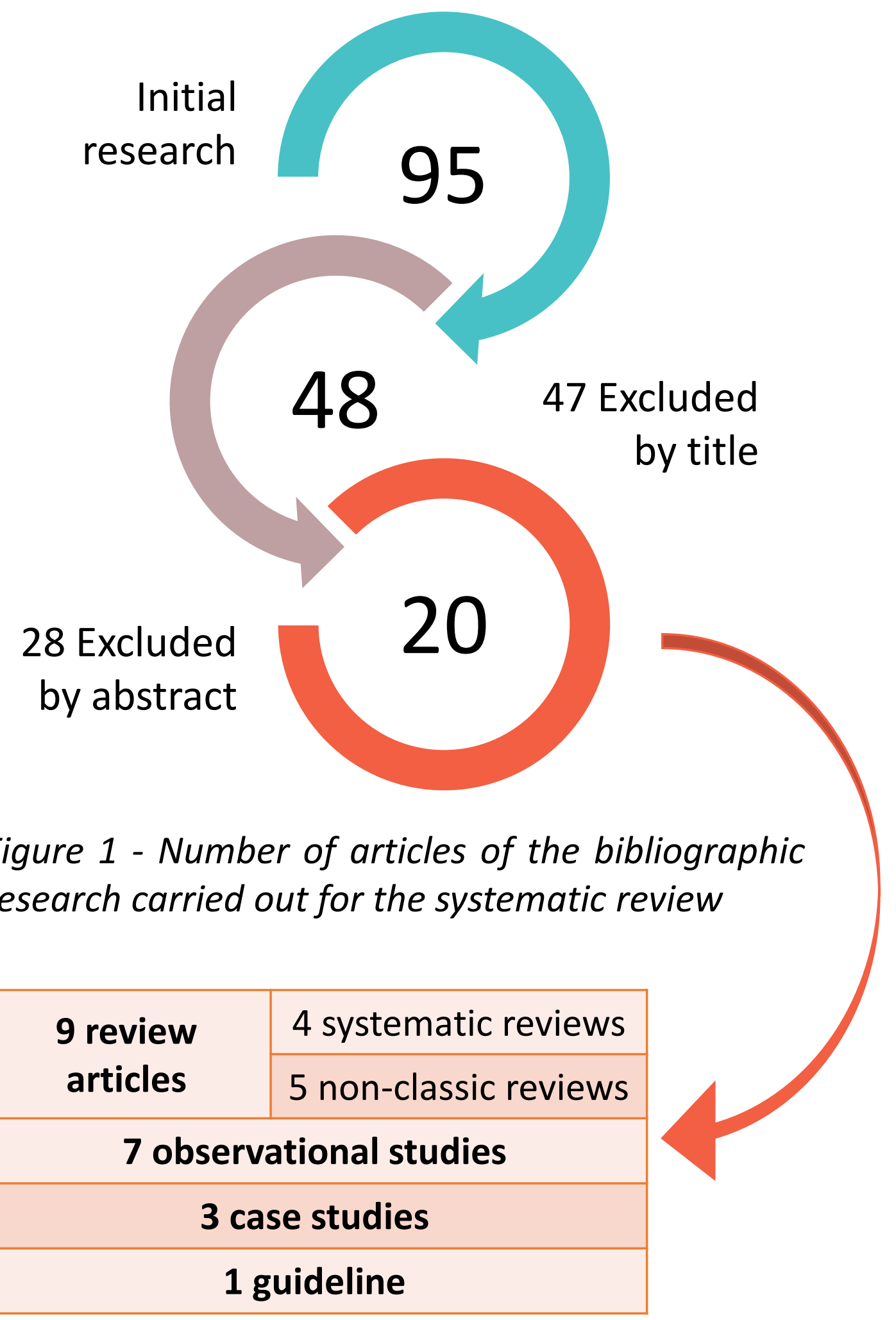

The research produced a total of 20 articles. Each review article was carefully evaluated according to the AMSTAR 2 criteria.

\section{Results}

The review showed few articles published about the evaluation of Chronic Pain. There aren't adequate scales for PHC's:

$\checkmark$ The most used methods of evaluation of pain are the Visual Analog Scale (VAS) and the Numerical Pain Scale (NPS).

$\checkmark$ To evaluate neuropathic pain the most used method was the DN4 questionnaire.

$\varnothing$ About evaluation of anxiety and depression in these patients, the articles selected presented great disparity and it wasn't possible to determine the best evaluation scale.

\section{Conclusion}

This protocol is indispensable to clinical practice because, without it, it isn't possible to have a 360 o view of the patient with pain. It can be replicable on any $\mathrm{PHC}$ and be a vital component for improving the quality of life of individuals suffering chronic pain.

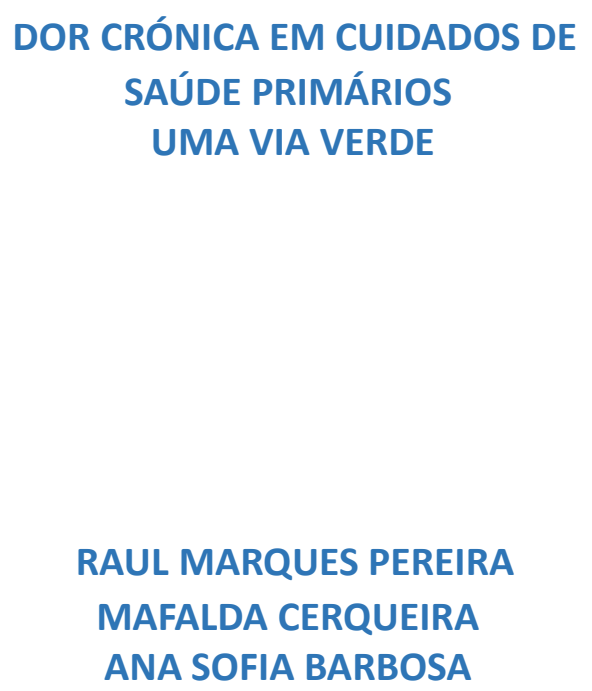

Figure 2 -Chronic pain assessment protocol Cover

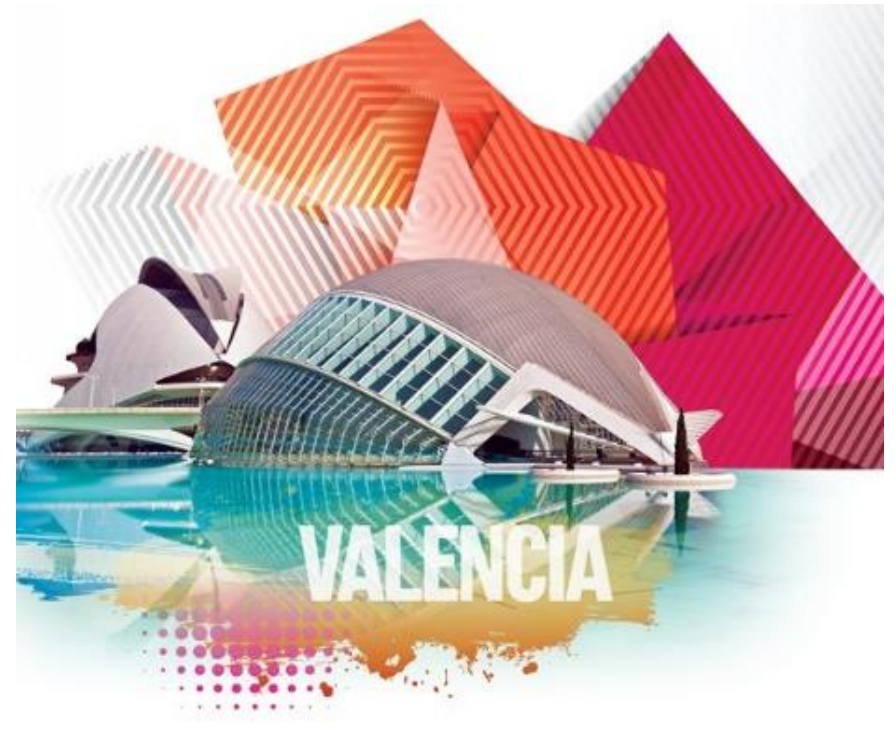

